\title{
ANALYSIS OF THE COMMUNITY DIAGNOSIS PROGRAM CONDUCTED BY DEPARTMENT OF COMMUNITY MEDICINE OF NOBEL MEDICAL COLLEGE BIRATNAGAR
}

\author{
Buna Bhandari, Mahesh Bhattarai, Rajiv Ranjan Karna and Nilam ar Jha
}

\begin{abstract}
Introduction: Community diagnosis program is a comprehensive assessment of health status of an entire community in relation to its social, physical \& biological environment.
\end{abstract}

Objectives: to know the students' feedback about the each component of Community Diagnosis Program (CDP) and to identify the knowledge and skills learnt by the students.

Methodology: A total 294 students from MBBS (219), BDS (50) and B Sc Nursing (25) students were posted for the CDP in different years for various activities in the field. The students were asked to give their feedback by the help of pre tested semi-structured questionnaire given to them. The data were analyzed in terms of percentage by using SPSS.

Results: Ninety four percent of the students were clear about objectives of CDP, $63 \%$ of them thought duration of CDP were adequate and household survey was given first rank (74.1\%) as very useful, followed by focus group discussion (69\%) and social mapping (57.1\%). The knowledge and skills learnt by the student during this posting were data collection, compilation, analysis and interpretation of data, communication skills, handling of the problem, cooperation and coordination among friends, understanding of the community, health status of community, cultural and behavioral aspects of community, respect towards other culture and beliefs, interaction with the community people.

Conclusion: Overall students rated CDP as a very good exposure for them in community setting. They have found it as interesting, relevant, memorable and very useful to increase their practical understanding of the community which will be very fruitful for them in future as a health professionals.

Key words: Community diagnosis program, feedback, Health, Socioeconomic status, Community

\section{Introduction}

According to WHO definition, community diagnosis is "a quantitative and qualitative description of the health of citizens and the factors which influence their health. $t$ identifies problems, proposes areas for improvement and stimulates action". ${ }^{1}$ Diagnosis of the community is reached from conclusions drawn from the data analysis. $t$ should preferably comprise three areas: health status of the community, determinants of health in the community, potential for healthy city development. $t$ is important to realize 
that Community Diagnosis is not a one-off project, but is part of a dynamic process leading to health promotion in the community. Therefore community diagnosis should be conducted at regular intervals. ${ }^{2}$ As for the community data, it can be collected by conducting surveys through self-administered questionnaires, face to face interviews, focus groups and telephone interviews. ${ }^{3}$

Community Diagnosis Program (CDP) is concurrent field program for MBBS student with BDS and B.Sc. Nursing students. For MBBS they will go for Community diagnosis in third semester whereas for nursing and BDS students in their first year. They go in village for 2 weeks. This program is being conducted to provide early exposure of the students to the community, a better understanding about health problems and the impact of socioeconomic status and culture on health and disease. The students perform followings activities in the field to fulfill the objectives. nteraction with village leaders to know about the village and obtain permission to conduct community diagnosis program, Social-mapping the area and house to house survey with structured questionnaire, Focus Group Discussion, Health education session, Health exhibition, Health check-up camp, Report presentation to the villagers, Report presentation at the college level and Data analysis and Report Preparation. ${ }^{4}$ A shift in the location from class room to clinical and community setting whenever feasible will enhance the extent of interaction. The undergraduate medical students are taken to the community during orientation program of their course to make them familiarize with local socio-cultural structures and norms. ${ }^{5}$ This study aims to know the students' feedback about the each component and duration of the program, to identify the knowledge and skills learnt by the students and to get suggestion from the students for improvement of the CDP.

\section{Methodology}

This descriptive cross-sectional study was conducted in Nobel Medical College Biratnagar from the Community Medicine department. A total 294 students from MBBS (219), BDS (50) and B Sc Nursing (25) students were posted for the CDP in different years (2010 to 2012) in different places. The CDP is of two weeks duration. After two days of orientation classes and preparation of the questionnaire, the students are taken for concurrent field visit for 10 days. After 10 days of busy field activities like social mapping, village leader meeting, data collection, focus group discussion, presentation at village level the students were back to the institute for the preparation and presentation of report. The students were asked to give their feedback with the help of pre- tested semi structured questionnaire. The data were analyzed by using SPSS where frequency and percentages were calculated. The CDP was supervised by the teachers from community medicine department. Verbal consent was taken from the participants before conducting the study.

\section{Results}

After analyzing the data of 294 students' view regarding the community diagnosis field program conducted by community medicine 
department various results were found. Regarding the objectives of the program, 94\% of the students said objective of CDP was clear whereas $6 \%$ said not clear to them.

(Fig 1).

Fig 1: View of students regarding objective of CDP

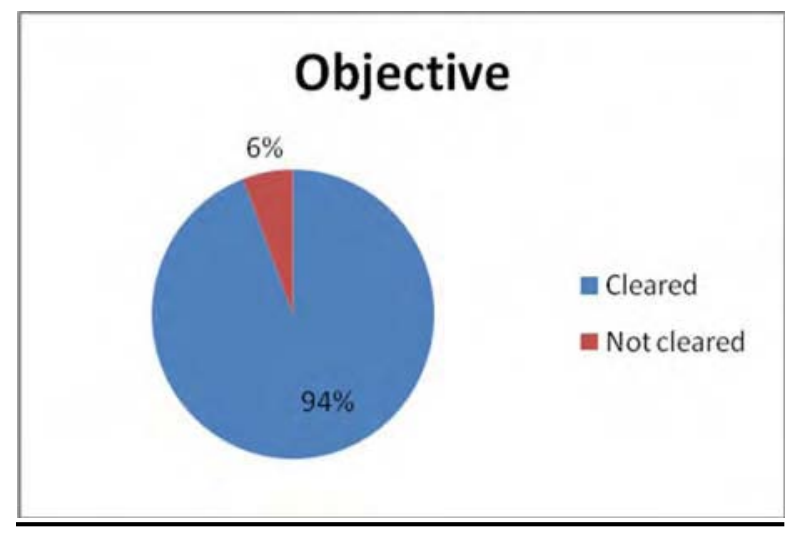

Similarly about duration of the study $63 \%$ of them thought that it was adequate, $36 \%$ of them thought that it was short and $1 \%$ thought it was long. (Fig 2)

Fig: 2 View of students regarding time duration of CDP

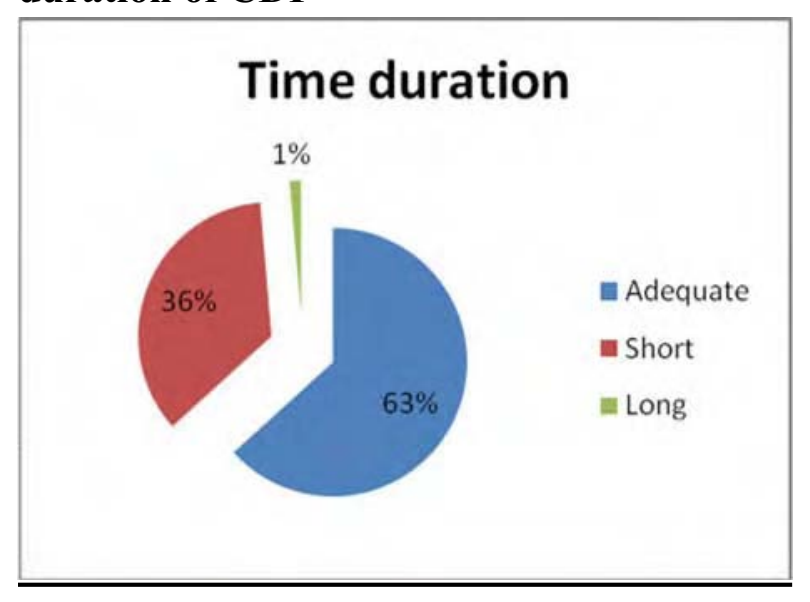

n Community Diagnosis Program various activities were conducted. According to them, the house to house survey was given first rank (74.1\%) as very useful, followed by focus group discussion (69\%) and social mapping (57.1\%) whereas 51\% said village leader meeting and $50 \%$ report presentation at college level, Health camp (46.6\%) as very useful. Most of the students said that report preparation (51\%) and community level presentation (52\%) and health exhibition (48\%) was useful which is shown in Table 1

Table 1: The rank of field activities of CDP $(\mathrm{N}=294)$

\begin{tabular}{lllcl}
$\mathbf{S}$ & \multicolumn{1}{|c}{ Activities } & $\begin{array}{c}\text { Less } \\
\text { useful }\end{array}$ & \multicolumn{1}{c}{ Useful } & $\begin{array}{c}\text { Very } \\
\text { Useful }\end{array}$ \\
$\mathbf{1}$ & House to house survey & $7(2.4)$ & $69(23.5)$ & $218(74.1)$ \\
$\mathbf{2}$ & Focus group discussion & $9(3.1)$ & $82(27.9)$ & $203(69)$ \\
$\mathbf{3}$ & Social mapping & $20(6.8)$ & $106(36.1)$ & $168(57.1)$ \\
$\mathbf{4}$ & Village leader meeting & $18(6.1)$ & $126(42.9)$ & $150(51)$ \\
$\mathbf{5}$ & $\begin{array}{l}\text { Report presentation at } \\
\text { College level }\end{array}$ & $14(4.8)$ & $133(45.2)$ & $147(50)$ \\
$\mathbf{6}$ & Health Camp & $37(12.6)$ & $120(40.8)$ & $137(46.6)$ \\
\hline $\mathbf{7}$ & Health exhibition & $16(5.4)$ & $141(48)$ & $137(46.6)$ \\
\hline $\mathbf{8}$ & Report preparation & $9(3.1)$ & $149(50.7)$ & $136(46.3)$ \\
\hline $\mathbf{9}$ & $\begin{array}{l}\text { Report presentation at } \\
\text { Community level }\end{array}$ & $21(7.1)$ & $154(52.4)$ & $119(40.5)$
\end{tabular}

Fig 3 illustrates the overall rating of CDP given by the students, most of them $44 \%$ said it was very good followed by excellent $25 \%$ and satisfactory $31 \%$.

Fig 3: Rating of CDP given by the students

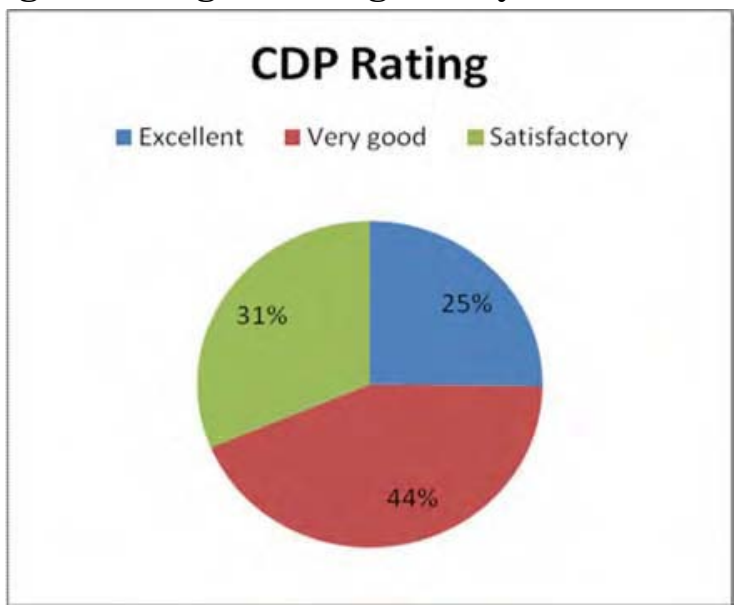

The knowledge and skills learnt by the student during this posting were data collection, compilation, analysis and interpretation of data, communication skills, 
handling of the problem, cooperation and coordination among friends, understanding of the community, health status of community, cultural and behavioral aspects of community, respect towards other culture and beliefs, interaction with the community people. Two important suggestions provided by the student for the improvement of this program were increased number of supervisor is needed to handle the large number of students, proper planning and adequate resources is needed for conducting the health camp at the end of CDP.

\section{Discussion}

Community Diagnosis Program is conducted for two weeks from the community medicine department for helping the students to understand the community setting better. After completion of CDP students were asked to give feedback of the program by filling self administered semi structured questionnaire. Ninety four percentages of students were cleared about the objectives of the study which shows that the students who were participated in the program had clear understanding about the objective of the program. The period of the CDP and usefulness of the posting were very satisfactory. They rated CDP as very useful. The rating regarding the usefulness of the activities of CDP showed House to house survey (74.1\%), Focus Group Discussion (69\%), Social mapping (57.1\%) were the best three activities liked by our students whereas study conducted in BPK HS showed Health checkup (96.1\%), health education (95.9\%), health exhibition (95.7\%) programs were the best. ${ }^{4}$ This shows students want to do the survey which increase their interaction and data collection skills as well as improve the skills working in the community. Most of the students (44\%) rated the CDP as a very good experience for them to understand the community. The knowledge and skills learnt by the student during this posting were data collection, compilation, analysis and interpretation of data, communication skills, handling of the problem, cooperation and coordination among friends, understanding of the community, health status of community, cultural and behavioral aspects of community, respect towards other culture and beliefs, interaction with the community people which were somehow similar to the study conducted in BPK HS. ${ }^{4}$ For the successful completion and implementation of the CDP efforts and commitment of the management, faculties as well as students are needed. Feedback obtained from the students after the each year of posting has been very positive. The students have rated the CDP as interesting, relevant, memorable and very useful. Community based education is associated with efforts to involve students and, more generally, educational institutions in national development and to combine theory with practice. Almost all countries have community based educational programs in which all types of social system and all levels of development are represented. However, they have been most successful in developing countries because of the benefit derived from the services of the students to the needy community and ultimately to the country. ${ }^{4}$

\section{Conclusion}

Overall students rated CDP as a very good exposure for the students of MBBS, BDS and 
B. Sc Nursing as it increases their skills like coordination, communication, working in a team approach assessing the community problem and solving by using local resources. Moreover they have found it as interesting, relevant, memorable and very useful to increase their practical understanding of the community which will be very fruitful for them in future as a health professionals.

\section{Acknowledgement}

We would like to acknowledge all the students of $4^{\text {th }} \& 5^{\text {th }}$ Batch MBBS, $1^{\text {st }}$ Batch BDS and $2^{\text {nd }}$ batch B.Sc Nursing of Nobel Medical College Teaching Hospital and Research Center, Biratnagar, for their feedback about the program. We would also like to extend gratitude to Mr. Sant Lal, (Lab boy of community medicine department) for helping in data entry. Finally, special thanks goes to all the faculties of Department of Community Medicine.

\section{References}

1. World Health Organization. City Health Profiles: how to report on health in your city. $\mathrm{CP} / \mathrm{HS} \mathrm{T} / 94 / 01$ PB 02 . Available at: www.euro.who.int/document/wa38094ci.pdf

2. Basic Principles of Healthy Cities: Community Diagnosis. Department of Health service.www.chp.gov.hk, updated in April 2009

3. Garcia P, McCarthy M. Measuring Health: A Step in the Development of City Health Profiles.
EUR/ CP/HC T 94 01/PB03. Available at: www.euro.who.int/document/ WA95096GA.pdf

4. Jha N. CDP- a multi-professional approach Health Renaissance, January-April 2011; Vol 9 (No.1); 41-44

5. Jha N, Pokharel PK and Ghartichhetri P,Yadav BK, Paudel IS, Niraula SR, Chapagain ML. Community Based Education: An Experience from Nepal. Journal of Nepal Medical Association 2002;41: 508-513.

Address for correspondence: Ms Buna Bhandari, Lecturer, Department of Community Medicine, Nobel Medical College, Kanchanbari, Biratnagar-5 